ÉGYPTE monde arabe

\section{Égypte/Monde arabe}

15-16 | 1993

Les crises soudanaises des années 80

\title{
Le développement comme outil de la paix : un rêve avorté
}

\section{Mohammed Ahmed Taisier}

\section{Q OpenEdition}

12 Journals

Édition électronique

URL : https://journals.openedition.org/ema/1107

DOI : 10.4000/ema. 1107

ISSN : 2090-7273

\section{Éditeur}

CEDEJ - Centre d'études et de documentation économiques juridiques et sociales

\section{Édition imprimée}

Date de publication : 31 décembre 1993

Pagination : $271-279$

ISSN : 1110-5097

\section{Référence électronique}

Mohammed Ahmed Taisier, «Le développement comme outil de la paix : un rêve avorté », Égypte/ Monde arabe [En ligne], 15-16 | 1993, mis en ligne le 08 juillet 2008, consulté le 07 juillet 2022. URL: http://journals.openedition.org/ema/1107 ; DOl : https://doi.org/10.4000/ema.1107

Ce document a été généré automatiquement le 7 juillet 2022.

Tous droits réservés 


\title{
Le développement comme outil de la paix : un rêve avorté
}

\author{
Mohammed Ahmed Taisier
}

\section{NOTE DE L'ÉDITEUR}

Traduit de l'anglais par Iman Farag.

1 Tout au long des dernières décennies, les notions de "paix » et de "développement » se sont fortement ancrées dans le discours politique soudanais. Avec chaque changement de régime, plus particulièrement depuis 1964, les déclarations politiques ont affiché, par un biais ou un autre, leur attachement à la réalisation de l'un de ces objectifs, voire des deux. Pourtant, la réalité est demeurée en deçà de la rhétorique. A croire que les élites au pouvoir cherchaient davantage à tirer parti du surcroît de légitimation que pouvait leur donner la charge forte et positive de ces notions, que de leur réalisation elle-même. Il est rare que leur usage se soit accompagné d'une perception claire des mesures et des politiques concrètes que supposait leur mise en œuvre. Rare, également, est la réflexion sur les articulations possibles entre paix et développement.

2 Au mieux, la pratique politique reflétait une grande confusion et chaque tendance avait sa propre conception de ce à quoi peuvent renvoyer les termes de "paix» et « développement ». Bien que les régimes politiques successifs se soient tous engagés à œuvrer au développement, le Soudan fait toujours partie - et de longue date - des pays les moins avancés. Et bien que ces régimes aient tous, depuis 1955, déclaré leur attachement à la paix, le pays est toujours miné par la guerre civile et les écarts entre discours et pratiques vont croissant. Le conflit armé, le sous-développement économique, politique, et social ont rapidement ébranlé le projet national d'une « unité dans la diversité »; d'un Soudan "trait d'union entre l'Afrique et le Monde arabe »; d'une nation "prospère " capable d'assurer ses besoins, ceux de ses voisins et de devenir le "grenier du monde». Le Soudan, qui devait figurer comme principal 
producteur de céréales dans la région, en est devenu incapable, paralysé par ses difficultés économiques. C'est au cœur de ce scénario cauchemardesque que s'élabore la dialectique du développement et de la paix...

3 Nous tenterons ici de cerner les facteurs responsables de l'échec des projets de pacification et de développement au Soudan depuis son indépendance, en 1956. Cela nous amènera à évoquer les conceptions qu'ont eues les différentes forces politiques des notions de paix et de développement, tant sur le plan historique que contemporain. La question posée est en définitive celle de la nature de l'État post-colonial soudanais.

Concepts et acceptions

4 Telle qu'elle est employée par les praticiens, la notion de développement est difficile à cerner. Un simple échantillonnage des définitions montre qu'il y en a autant qu'il y a d'auteurs. Cependant, quand bien même cette notion résisterait à la définition, cela n'interdit ni son emploi ni sa concrétisation à travers des pratiques. Il n'est pas un seul des pays occidentaux ou industrialisés qui n'ait ses départements ou ses ministères pour le développement local, régional ou international. Il en est de même pour les pays du tiers-monde qui, s'ils veulent être pris au sérieux, doivent consacrer au développement une part importante de leur structure ministérielle. Les Nations Unies ont leurs agences de développement, la Banque mondiale contient dans son titre officiel la notion de développement. Les praticiens du développement se comptent par milliers et des sommes considérables sont versées à cette fin. Sans savoir en quoi consiste le développement, nous savons qu'il existe et qu'il constitue un objectif à atteindre.

Il n'en a pas toujours été ainsi. Le développement comme action volontaire et intentionnelle est une notion moderne. Avant de devenir pratique, il était un fait. Et comme le suggère l'étymologie même du terme, il se "déroulait", se "déployait». Pourtant, l'acception moderne de la notion de développement comme action volontaire n'a pas mis fin au sens premier, " évolutionniste » si l'on peut dire. Tout porte à croire que la difficulté de définir le développement provient de la collusion de ces deux acceptions, qui s'accompagnent plutôt que de se substituer l'une à l'autre.

6 L'on sait également qu'un développement viable ne peut être imposé par le haut, comme le prouve l'échec inéluctable d'expériences développementalistes qui ont tenté de s'imposer aux masses. Dans le contexte des États post-coloniaux s'impose également ce constat: plus grand a été le respect de la démocratie - même dans sa définition restreinte - plus élevés ont été les taux de croissance et plus adéquats les modèles de croissance. C'est par leurs conduites participatives que les forces sociales parviennent à négocier un nouvel équilibre entre environnement physique et environnement social ainsi qu'entre elles-mêmes.

7 Cette "philosophie du développement » nécessite un nouveau regard sur les cultures locales. Il est certain que celles-ci occupaient une place centrale dans la question du développement, mais elles l'ont perdue pour plusieurs raisons dont, principalement, la prédominance d'une rationalité dite "technique». Les cultures locales ne sont pas de simples dépositaires historiques d'une sagesse ancestrale des peuples et de leurs traditions; elles sont également expérience, savoir et savoir-faire. Tous ces attributs devraient faire partie de l'équation du développement. En d'autres termes, c'est de la réalisation d'une synthèse réussie entre les données de la culture locale et les impératifs du développement, que dépend la réussite de celui-ci. Cette culture locale 
est multiple; l'accepter en tant que telle, c'est admettre, reconnaître et respecter l'altérité, les coutumes et manières de faire de toutes les communautés.

8 Ce sont ces mêmes éléments que l'on retrouve à la base de tout état de paix sociale. Celle-ci ne peut être perçue uniquement comme absence d'état de guerre ou de belligérance. Une société entre en état de guerre interne quand l'un ou plusieurs de ses groupes, tentent de réaliser des objectifs perçus comme menaçants par un ou plusieurs autres groupes. Quelles que soient les racines de ces conflits internes ou leur conjoncture, ils renvoient à des différences socio-économiques ou socioculturelles.

Les expériences de la plupart des pays d'Afrique qui avaient conçu le développement comme moyen de résorption de ces conflits ont échoué, le plus souvent, du fait de la concurrence des intérêts et des aspirations socioculturelles. Cela dit, il nous faut rappeler que le volontarisme seul ne suffit pas quand il s'agit de mettre fin à ces types de conflits. Le développement en question devrait assurer aux Soudanais le minimum de pain quotidien et leur épargner les ravages de la guerre civile.

Le développement est souvent perçu comme une lutte entre tradition et modernité ; son essence se confondrait avec celle de la modernisation. Cependant, les faits sont là pour rappeler que modernisation et ethnicité ne sont pas forcément contradictoires ; voire que la modernisation intensifie la conscience et les conflits ethniques. Assimilé à la modernisation, le développement n'entraîne ni règlement des conflits ni croissance socio-économique équitablement répartie. Ni la paix ni le développement ne peuvent être perçus comme des valeurs abstraites indépendantes du contexte historique, des forces sociales en compétition et des réalités politiques et culturelles.

11 Etablir le lien entre problématique du développement et conflit interne permet de résoudre les deux dilemmes, dans la mesure où cette problématique engage la participation populaire. Les réalités politiques africaines montrent que moins les régimes sont ouverts à la participation politique, plus leur survie est précaire et leur situation économique difficile.

Idéologie et pratiques du développement

12 L'étude des problèmes de développement au Soudan soulève plus de questions qu'elle ne donne de réponses. Si les faits relatifs aux politiques de développement ont été soigneusement inventoriés et relevés, il y a peu d'éléments sur les raisons de tel ou tel choix en matière de développement et les couches sociales qui en ont été les bénéficiaires. Parallèlement, les conséquences négatives des politiques de développement n'ont pas fait l'objet d'analyses. Une des raisons de celte carence est l'hypothèse implicite que partagent nombre de travaux qui tendent à percevoir l'État comme expression d'un « intérêt national supérieur ", quand bien même cette notion reste vague. Dans ce cadre, l'État étant garant de l'intérêt dit national, le développement ne peut que se dérouler, s'étendre, dans le sens prométhéen et naturaliste que l'on donne à cette notion.

13 Les politiques étatiques ne sont le fait ni d'un ministre ni d'un département d'État, et rien ne suggère qu'elles aient été adoptées «pour le bien de tous » et «en faveur de tous ». L'État n'est ni une émanation divine, ni une institution cohérente et unifiée, qu'il faille vénérer ou haïr en elle-même. A l'instar des autres institutions, il est le produit de rapports entre groupes sociaux et individus ayant une place déterminée dans le cadre de rapports de production. L'État a cela de particulier qu'il reflète ou condense la division fondamentale des intérêts dans une société. Ainsi, les liens entre choix politiques et programmes de développement ne sont-ils pas aléatoires. Bien au 
contraire, ces liens sont ancrés dans les intérêts des divers groupes sociaux et économiques. Aussi n'est-il pas possible de débattre des stratégies de développement indépendamment des intérêts politiques.

C'est autour de l'agriculture que les stratégies de développement soudanaises se sont concentrées dans la mesure où le secteur agricole emploie plus de $85 \%$ de la maind'œuvre, produit environ $40 \%$ du PNB, constitue $90 \%$ des ressources en devises et $90 \%$ des exportations. Outre le fait qu'elles affectent de manière directe la vie quotidienne des Soudanais, les politiques agricoles sont également un des principaux terrains où s'exercent le poids et les intérêts des forces socio-économiques dominantes.

Les initiatives de développement touchant à ce secteur se sont concentrées principalement dans les régions de la Gezira, du Nil Bleu et du Nil Blanc. Les autres régions n'ont fait l'objet d'attention que dans la mesure où leur rendement agricole permettait une accumulation des ressources de l'État et des groupes dominants bénéficiaires. L'infrastructure des services sociaux, sanitaires et éducatifs s'est quasiment limitée aux zones urbaines liées à la capitale, point de transit obligé des produits agricoles destinés à l'exportation, alors que les habitants de l'arrière-pays, livrés à la sous-nutrition et à la pauvreté, ne recevaient de l'État aucun soutien financier. Leurs difficultés se sont aggravées avec les migrations croissantes des jeunes et des travailleurs à la recherche de meilleures chances d'éducation et d'emploi.

Les populations du Sud-Soudan ont connu pire. Partageant la détérioration socioéconomique commune à toutes les zones rurales, elles ont dû subir également une violence d'État qui s'est exercée sans ménagement. C'est ainsi qu'entre 1956 et 1972, et à nouveau depuis 1983, les ébauches de plans de développement agricole ou autres ont échoué. Des dizaines de milliers de Soudanais se sont dirigés vers les pays voisins à la recherche de refuge ou d'emploi. Ceux qui se sont déplacés vers les zones urbaines du pays ont dû affronter la compétition entre migrants dans leur recherche d'emplois saisonniers ou irréguliers.

17 L'instabilité des régions du sud a fourni au gouvernement soudanais une bonne excuse pour " geler » toute velléité de projets de développement en faveur des populations locales. Dans les faits, qu'il s'agisse des 17 premières années de l'indépendance soudanaise ou de la période ayant suivi l'année 1984, bien peu de choses ont été faites pour améliorer le sort des populations. En revanche, l'État a concentré ses efforts vers les objectifs de type militaire, perpétuant la violence, menaçant la paix et coupant court à tout développement.

Le problème économique soudanais est souvent perçu en termes de dualisme et de partage contesté des ressources entre secteur moderne et secteur traditionnel. A notre sens, ce dualisme constitue plus un symptôme qu'il n'est la cause du sousdéveloppement économique, des limites de la croissance économique et de la détérioration généralisée des conditions socio-économiques que subit la majorité de la population.

19 C'est qu'il existe un autre dualisme plus ancré et plus insidieux. Il tient tout d'abord aux caractéristiques fondamentales du pouvoir soudanais. Tout au long de la période qui a suivit l'indépendance, les forces dominantes et l'élite au pouvoir n'ont pas réussi à élaborer un programme de développement économique ayant des objectifs clairs et précis. Ainsi l'État soudanais n'a-t-il jamais eu une politique à long terme réellement définie, ni un engagement relatif aux modes de production et à la propriété des moyens de production. Pas plus qu'il n'a décidé si le développement devait s'appuyer sur une 
technologie moderne, une technologie intermédiaire ou demeurer fondée sur le recours à une main-d'œuvre intensive. Bref, l'État soudanais s'est révélé incapable de formuler une "philosophie du développement». Par ailleurs, les décisions ont été prises par une faction au sein des groupes dominants, imposées par le haut sans la moindre participation significative de la part de la société. La bureaucratie est restée enfermée dans ce qu'on peut identifier comme des pratiques et des attitudes historiquement définies par le mode de structuration du pouvoir et de l'administration coloniale.

Au Soudan, tout comme dans d'autres colonies britanniques, un plan de développement quinquennal avait été introduit pour la période 1946-1951, suivi d'un second en 1951-1956. Ces plans, ainsi que les cadres qui ont servi au suivi de leur déroulement, ont été autant d'incubateurs pour les idées et pratiques des puissances économiques postcoloniales. Depuis l'indépendance en 1956 et jusqu'en 1961, la planification à long terme a été interrompue. Les régimes de cette période se sont en revanche basés sur des documents intitulés " Nouvelle série annuelle de projets ", qui se réduisaient à de curieuses propositions dont la plupart n'avaient rien de nouveau. Plusieurs d'entre eux ont été copiés sur la période coloniale et quelques-uns dictés sous la pression des conditions politiques. L'indépendance n'a entraîné aucun changement ni dans l'idéologie ni dans les opérations de planification du développement.

C'est que l'ensemble des politiques de l'État soudanais n'a pas connu de changements malgré l'indépendance. Plus de trois décennies après l'indépendance formelle, le Soudan reste dépendant en matière de biens de consommation et importe tant pour combler ses besoins alimentaires que pour se fournir en produits finis. En termes généraux, les plans économiques post-coloniaux ont maintenu en place les mécanismes d'appropriation du surplus basés sur la subordination de la paysannerie et l'accaparement de la production agricole. Cela explique aussi que l'autoritarisme d'État soit demeuré inchangé et qu'aient disparu diverses formes de participation populaire et des droits fondamentaux. Organisé à l'origine par le pouvoir colonial, imposé et maintenu pour soumettre la production de l'économie nationale aux impératifs de l'échange avec les marchés internationaux, l'État est demeuré inchangé après l'indépendance.

22 Les deux principaux partis constitués dans la moitié des années 40 n'avaient aucun programme et ne recevaient pas de cotisations de leurs membres. L'aristocratie confrérique leur a fourni non seulement, le soutien matériel et financier mais, plus important encore, son capital social et son poids symbolique ont garanti la pérennité des tendances chauvines au sein des Soudanais. Ces partis n'étaient basés sur aucune sollicitation du soutien des masses à travers l'expression des attentes et des revendications. Bien au contraire, conçus dans les limbes de l'aristocratie religieuse, ils sont restés fidèles, dans leurs conduites politiques, à leur base de départ.

En d'autres termes, les partis dominants n'ont jamais constitué une réelle menace ni pour l'ordre colonial ni pour les rapports sociaux néo-coloniaux. Ils cherchaient à obtenir, des changements constitutionnels sans pour autant permettre aux masses de renforcer leur autonomie d'action et leur organisation, tant sur le plan économique que sur le plan culturel. Leur préoccupation principale était le maintien de la stabilité, de l'ordre et de la loi. Cette volonté de préserver le statu quo avait pour origine une grande appréhension du changement. Il est vrai que toute tentative de restructuration de l'ordre politique et social risquait de mettre fin au pouvoir, aux privilèges, et aux 
intérêts des groupes dominants, retranchés derrière et entretenus par des rapports économiques de type colonial.

En ce sens, l'indépendance était bien une des étapes de la formation sociale soudanaise et non un changement structurel au sein de cette formation. Aussi les politiques économiques et les stratégies de développement - quand elles ont existé - se sontelles préoccupées du contrôle, de la distribution et de la consommation des ressources et des produits nationaux en fonction des intérêts déjà établis des forces politiques dominantes. Une des caractéristiques de la période de l'indépendance est la fréquence des intrigues politiques au sein du bloc au pouvoir et la concentration des efforts de l'élite politique dans l'accaparement du pouvoir. Mobilisés par leurs luttes intestines, les gouvernements successifs ont fait bien peu de choses pour affronter les problèmes politiques et économiques liés au sous-développement.

Les partis politiques n'avaient pas conscience des besoins nationaux urgents. Pour la plupart, saisir la dynamique du sous-développement ou les nécessités du développement national dépassait à la fois leur horizon mental, leurs intérêts et leurs compétences. Leur perception des réalités socio-économiques et nationales sont restées intuitives, fragmentées et limitées. Etant donné cette orientation du leadership politique et le peu de cas qu'il faisait des intérêts immédiats de reconstruction nationale, la bureaucratie dans son ensemble n'a pas perçu la nécessité de réorienter les appareils et les administrations d'État en les éloignant de leur visée coloniale ou de formuler une politique de développement national correspondant aux besoins et aspirations populaires.

Ces circonstances ont accéléré la désintégration nationale, précipitant le pays dans la guerre civile. Les échecs décrits plus haut, les carences dans le fonctionnement de l'appareil d'État post-colonial, les séries de promesses jamais réalisées, ces «multitudes d'engagements non respectés » à propos desquels écrit Abel Alier, tout cela a déclenché la guerre civile, la violence politique et la répression qui ont dévasté le sud pour de nombreuses années.

Pour comprendre les raisons pour lesquelles cette partie du pays est restée exclue des plans de développement, il convient de revenir sur quelques points. Durant la période coloniale, l'activité économique s'est intensifiée dans les régions productrices de coton entre les deux Nils, tout au long des routes traditionnelles du commerce avec le nord et l'Egypte et des enclaves portuaires sur la mer Rouge. Les régions de l'extrême nord, de l'est et de l'ouest, éloignées des pôles économiques et des voies de communication importantes, et peu touchées par les formes modernes de commercialisation, n'ont eu droit qu'à un bénéfice économique marginal.

Les régions du sud ont partagé ce sort, aggravé sans doute par les politiques administratives de l'époque post-coloniale et le caractère anarchique des stratégies de développement dans les années qui ont suivi directement l'indépendance. Le Closed District Ordinance (adopté durant la période coloniale) a étouffé la région et a considérablement affaibli ses liens économiques avec l'ensemble du pays. En l'absence de dynamique économique privée ou d'entreprises importantes, ni les forces socioéconomiques, ni les factions qui composaient le groupe au pouvoir n'ont accordé d'intérêt à la région et à la recherche de la paix. Elles pouvaient continuer à ignorer les aspirations populaires et les fondements réels du conflit et à traiter la crise et, par la suite, la guerre civile comme une mutinerie ou un problème militaire à régler par les armes. 
29 Nous avons tenté de souligner ici quelques-unes des corrélations directes entre paix et développement, à travers l'évocation de faits largement évidents. Nous avons mis également en valeur le fait que, depuis l'indépendance, seules des demi-mesures ont été adoptées pour débarrasser l'État de son héritage colonial, restructurer ses institutions, redéfinir et orienter ses politiques de manière compatible avec les objectifs de reconstruction nationale. De tels changements s'imposaient dans la mesure où cet État avait été conçu et mis en place pour le maintien de l'ordre colonial. En tant que tel, il était inconcevable qu'il prit en charge un Soudan indépendant et ses objectifs nationaux, sociaux, économiques et politiques.

L'État post-colonial est resté inchangé et n'a pu ainsi assimiler les changements qui ont affecté la formation sociale soudanaise, voire même arbitrer avec succès les intérêts des forces sociales les plus importantes. Cet échec et les contradictions sociales qu'il a engendrées ont rendu l'État de plus en plus insensible aux aspirations de la population et incapable de satisfaire ses besoins élémentaires. Il n'est pas étonnant que, non seulement les politiques post-coloniales soient mises en échec, mais aussi qu'elles constituent la plus forte menace à l'unité nationale en rendant encore plus difficile le dépassement des problèmes sociaux, économiques et politiques.

Un des obstacles les plus important à la paix réside dans ce qu'on pourrait identifier comme une profonde "apathie » vis-à-vis de la guerre civile en dehors des zones de belligérance, qu'il s'agisse des pertes de vies humaines, de la destruction, de l'épuisement des richesses nationales ou des chances de développement manquées. Il reste à développer un sens de l'urgence politique - en finir avec cette guerre - tant au sein du corps politique que dans l'ensemble de la société. L'indifférence de l'opinion à l'égard de la guerre, le sentiment qu'elle se déroule sur une autre planète ou dans des contrées fort lointaines, retarde et amoindrit les chances de parvenir à un règlement durable du conflit. Il s'agit donc de renforcer la prise de conscience populaire quant aux effets dévastateurs du conflit sur la vie quotidienne, du niveau de vie et de l'ensemble du fonctionnement social. Bref, de mettre en évidence que les intérêts de tout un chacun sont directement et concrètement liés à la fin de cette guerre.

Les expériences précédentes en matière d'instauration de la paix depuis 1985 montrent que l'échec est fortement lié à l'incapacité à inscrire dans les mentalités cette corrélation entre paix et développement. Il importe donc de formuler les modalités de cette articulation entre les deux objectifs. Ne pas élaborer l'agenda de la paix sur de telles bases n'aboutira qu'à reconduire le sous-développement et à rendre encore plus lointain le rêve d'un Soudan prospère, uni, riche de son héritage pluri-culturel.

\section{INDEX}

Mots-clés : Soudan 
AUTEUR

MOHAMMED AHMED TAISIER

Université de Khartoum 